\title{
Temperature-dependent gaps in the half-filled Hubbard model on a triangular lattice
}

\author{
M. C. Refolio,* J. M. López Sancho, and J. Rubio \\ Instituto de Matemáticas y Física Fundamental, CSIC, Serrano 113 bis, 28006 Madrid, Spain \\ (Received 21 March 2001; revised manuscript received 3 July 2001; published 1 February 2002)
}

\begin{abstract}
The evolution of gaps in the one-electron density of states for the half-filled Hubbard model on a triangular lattice is studied as a function of both the temperature and the coupling constant (Hubbard $U$ ) using quantum Monte Carlo. The formation of gaps (or pseudogaps) at finite temperature allows us to distinguish between three regimes: (1) A strong-coupling Mott-Hubbard regime, characterized by a gap, which persists even at high temperatures; (2) a weak-coupling paramagnetic regime, characterized by the absence of a pseudogap at any finite temperature; and (3) an intermediate-coupling spin-density-wave regime, characterized by a pseudogap, which appears when $U$ is increased beyond a critical (temperature-dependent) value. The behavior of the $\sqrt{3}$ $\times \sqrt{3}$ adlayer structures on fourth-group semiconductor surfaces is briefly commented upon in the light of the above discussion.
\end{abstract}

DOI: 10.1103/PhysRevB.65.075114

PACS number(s): 71.15.Mb, 73.20.At, 75.30.Fv

\section{INTRODUCTION}

For many years the triangular lattice has been a subject of much interest due, mainly, to the frustrating effects its nonbipartite nature entails. These often lead to nontrivial ground-state degeneracies as in the antiferromagnetic (AF) spin one-half Ising model. ${ }^{1,2}$ The classical Heisemberg model on the two-dimensional (2D) triangular lattice with nearest-neighbor AF coupling and easy-axis exchange anisotropy is another example where frustration leads to a novel ground-state degeneracy. This system has attracted much attention especially since Anderson ${ }^{3}$ suggested the possibility of a resonating-valence-bond ground state for the spin one-half case. Simply, the quantum liquid of radomly distributed spin-singlet pairs could be an efficient way to overcome the frustration of the Néel state in the triangular antiferromagnet. One more important source of interest in these lattices is the well-known diversity and richness of physical phenomena displayed by most transition-metal compounds. ${ }^{4}$

The recent experimental observation of low-temperature insulating phases in some $\sqrt{3}$-adlayer structures on (111) $\mathrm{Si}$ and Ge surfaces has only fostered the interest in these 2D triangular lattices. Thus, whereas the $\sqrt{3}$ overlayers of $\mathrm{Sn}$ and $\mathrm{Pb}$ on $\mathrm{Ge}(111)$ are both metallic at a high temperature, their corresponding low-temperature counterparts are either metallic, as in the case of $\mathrm{Sn}^{5-7}$ or weakly insulating as in the case of $\mathrm{Pb} .{ }^{8-10}$ This latter system seems to go through some kind of reversible metallic-to-insulating transition whose precise nature is still controversial. ${ }^{11-13} \mathrm{~A}$ chargedensity wave has been invoked in the case of $\mathrm{Pb}$ (but not in the case of $\mathrm{Sn}$ ) as the driving force for the destabilization of the high-temperature phase, a conjecture not universally accepted. Related isoelectronic systems, on the other hand, such as the $\sqrt{3} \times \sqrt{3}$ adlayer of $\mathrm{Si}$ on $\mathrm{SiC}(0001),{ }^{14}$ or of $\mathrm{K}$ on $\mathrm{Si}(111)$ :B (Ref. 15) show a clear insulating behavior with a large gap and no phase transitions. These systems have been studied theoretically both within the local-density ${ }^{5,16,17}$ (LDA) and the Hartree-Fock ${ }^{18,19}$ approximations. Quite recently ${ }^{20}$ the $\mathrm{LDA}+U$ approach has been used to include strong on-site repulsions in $\mathrm{SiC}(0001)$.
In this paper we carry out a model study of the triangular lattice in order to explore some general questions any realistic theory should comply with. For instance, understanding the temperature behavior of the one-electron density of states (DOS) is essential to the development of a complete picture of these metal-insulator transitions. Hence we report the results of a quantum Monte Carlo (QMC) simulation of the half-filled Hubbard model on such a triangular lattice in the grand canonical ensemble. The one-electron Green's function is studied as a function of both the temperature and the coupling constant (Hubbard $U$ ). As the temperature is lowered, a pseudogap develops in the one-electron DOS for intermediate values of $U$. This pseudogap is accompanied by two weak peaks in the spin structure factor which signal the formation of a complex spin-density-wave (SDW) structure. For lower $U$, no gap at all is found even for low temperatures, the system remaining always paramagnetic. For higher $U$, on the other hand, a well-developed gap appears at any temperature, accompanied by a strong peak in the spinstructure factor. The system is then brought into a state very similar to the ground state of the triangular antiferromagnet (the three-sublattice model). We emphasize that these are not distinct phases, but only different regimes with smooth transitions among them, as characterized by the behavior of the one-electron DOS. Since the presence or absence of a gap or pseudogap is of fundamental importance in determining the properties of a system, we believe that this type of characterization is useful and can be of help in understanding the electronic properties of the more complex adsorption systems referred to above.

The paper is organized as follows: Section II recalls some of the basic properties of the triangular lattice as well as the Hubbard model in order to fix the notation. The resulting one-electron DOS and spin-structure factor are displayed and discussed in Sec. III and, finally, the paper closes with some concluding remarks in Sec. IV

\section{THE MODEL}

In the $\sqrt{3} \times \sqrt{3} R 30^{\circ}$ adlayer structures of $\mathrm{Sn}$ or $\mathrm{Pb}$ on $\mathrm{Ge}(111)$ at one-third coverage, each adsorbate sits on top of 


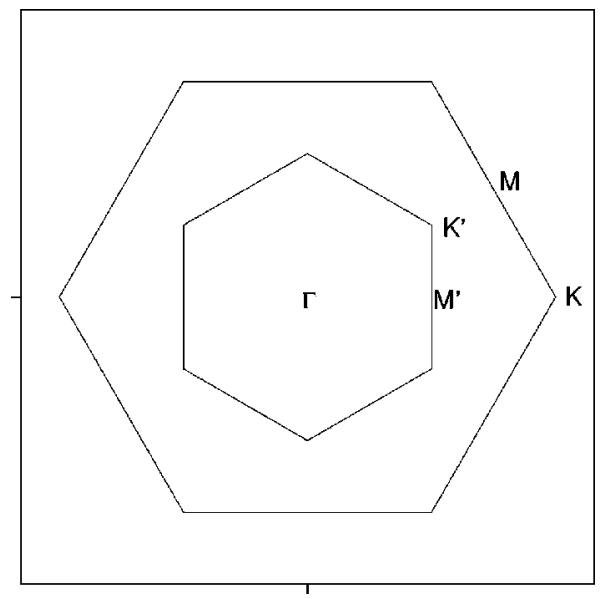

FIG. 1. Large (outer hexagon) and small (inner hexagon) surface Brillouin zones (SBZ) of the triangular lattice. Shown are the especial points $\Gamma, M^{\prime}, K, M$, and $K^{\prime}$ which delimit the contours $\Gamma K M \Gamma$ and $\Gamma M^{\prime} K^{\prime} \Gamma$ used in the text.

a triangle of Ge atoms. With just one unpaired electron per adsorbate, the overlayer is half filled and, therefore, metallic in the absence of electron-electron interactions. This overlayer can in turn be described as a $3 \times 3$ lattice of adsorbate triangles (the three-sublattice model) with three unpaired electrons per triangle and, therefore, again metallic in the absence of interaction. The corresponding surface Brillouin zones (SBZ) are the large and small hexagons, respectively, in Fig. 1. The adlayer $\sqrt{3} \times \sqrt{3}$ lattice has just one band in the large zone $\varepsilon_{k}^{0}$, which folds onto three bands in the small zone. Figure 2 shows these three bands, $\varepsilon_{k}^{0}, \varepsilon_{k}^{+}$, and $\varepsilon_{k}^{-}$, unfolded in the extended zone scheme in order to see the nesting properties. They are given by $[t=$ (hopping strength) $]$

$$
\begin{gathered}
\varepsilon_{k}^{0}=2 t \cos k x+4 t \cos \frac{1}{2} k_{x} \cos \frac{\sqrt{3}}{2} k_{y}, \\
\epsilon_{k}^{ \pm}=-\frac{1}{2} \varepsilon_{k}^{0} \pm t \sqrt{3}\left(\sin k_{x}-2 \sin \frac{1}{2} k_{x} \cos \frac{\sqrt{3}}{2} k_{y}\right) .
\end{gathered}
$$

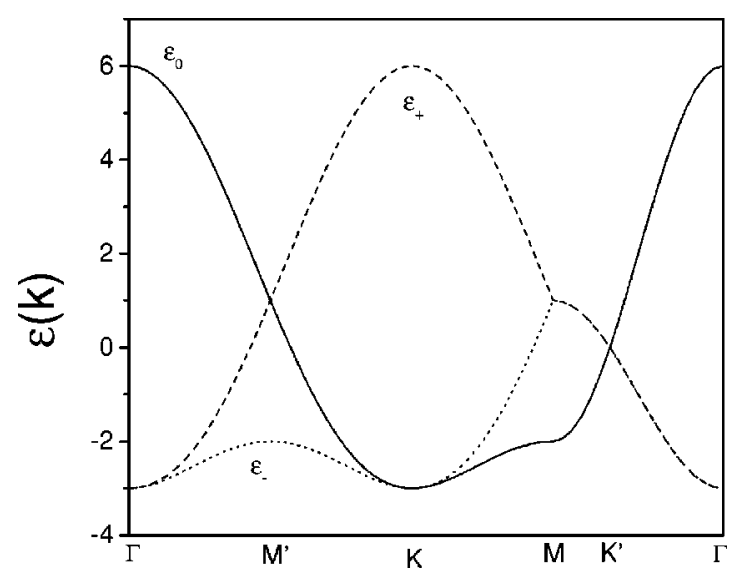

FIG. 2. Band structure of the triangular lattice for $U=0$ in the three-sublattice model. The three bands, $\varepsilon_{k}^{0}$ (main band) and $\varepsilon_{k}^{ \pm}$, are displayed along the $\Gamma K M \Gamma$ contour of the large SBZ in order to show the band crossings and nesting symmetry.

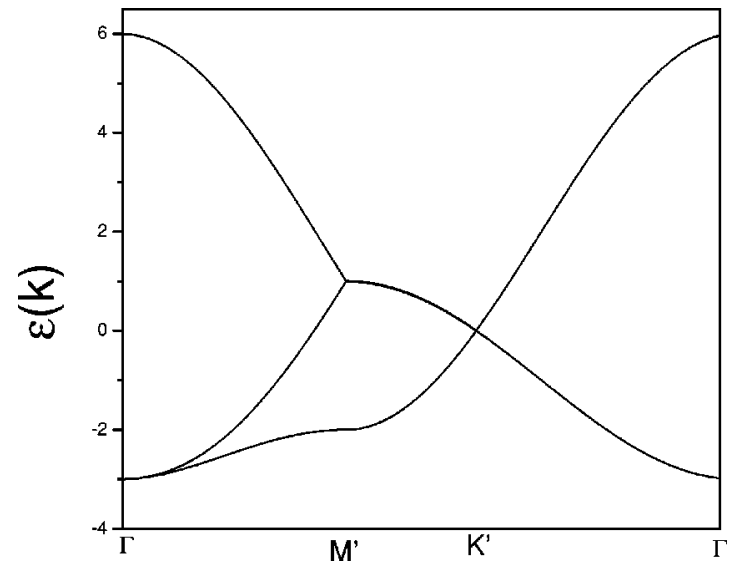

FIG. 3. Same as Fig. 2, but along the $\Gamma M^{\prime} K^{\prime} \Gamma$ contour of the small SBZ.

It is easy to see that $\epsilon_{k}^{ \pm}$are just $\epsilon_{k}^{0}$ for $k=\left(k_{x} \pm 2 \pi / 3, k_{y}\right)$. These bands cross at the points $M^{\prime}$ and $K^{\prime}$. The wave vector $K=(4 \pi / 3,0)$ turns out to be a nesting vector with the band folding around the $M^{\prime}=0.5 K$ point $\left(M^{\prime} K \rightarrow M^{\prime} \Gamma\right.$, band $\varepsilon_{k}^{+}$). Likewise $K M \rightarrow \Gamma M^{\prime}$ (band $\varepsilon_{k}^{-}$,). Figure 3, finally, shows the resulting band along the small SBZ contour. Notice that the $M^{\prime} K^{\prime}$ direction is obtained by folding the $M K^{\prime}$ portion of $\varepsilon_{k}^{0}$.

This is of no consequence for the interaction-free system at half filling, since the Fermi surface is not anywhere close enough to either the large or the small SBZ boundaries. When the interaction is turned on, however, the nesting symmetry may come into play, although weakly, at the points $M$ and $M^{\prime}$, closest to the Fermi surface (see Fig. 1 of Ref. 19). We shall see that, even at half filling, this is indeed the case for the spin-structure factor when $U$ is large enough.

In order to describe the interacting system, we adopt the Hubbard model, given by the standard Hamiltonian

$$
H=t \sum_{\langle i j\rangle s} c_{i s}^{\dagger} c_{j s}-\mu \sum_{i s} n_{i s}+U \sum_{i}\left[\left(n_{i \uparrow}-\frac{1}{2}\right)\left(n_{i \uparrow}-\frac{1}{2}\right)\right],
$$

where $t$ is the hopping strength, $U$ the on-site repulsion, and $\mu$ the chemical potential. The single sums run over all the $N \times N$ adlayer atoms and the symbol \langle\rangle means summation over nearest neighbors. As usual, $c_{i s}^{\dagger}$ creates, while $c_{i s}$ destroys, an electron of spin $s$ at site $i$ with occupation number $n_{i s}=c_{i s}^{\dagger} c_{j s}$. We take $t=0.055 \mathrm{eV}$ so as to start with a narrow adlayer bandwidth $(W=9 t)$ of around $0.5 \mathrm{eV}$ at $U$ $=0 . U$ is varied to cover different regimes of the triangular lattice and $\mu$ is adjusted so as to have always half filling. Recall that, unlike the case of bipartite lattices, $\mu=U / 2$ does not necessarily correspond to half filling since particle-hole symmetry does not hold in a triangular lattice even at half filling.

This Hubbard model is now simulated by the QMC approach in the grand canonical ensemble as initially developed by Blankenbecker et al. ${ }^{21}$ and supplemented by a discrete lattice version of the Hubbard-Stratonovich (HS) transformation by Hirsch. ${ }^{22}$ The whole approach has been 
explained at length by $\mathrm{Hirsch}^{23}$ and White et al. ${ }^{24}$ For an excellent review see Loh and Gubernatis. ${ }^{25}$ The lack of particle-hole symmetry alluded to above entails that the minus-sign problem of the Fermion determinant appears, even at half filling, in the triangular lattice for some Ising configurations of the HS field. These configurations usually give unphysical values for some physical quantities [e.g., level occupancies outside the $(0,1)$ interval]. This was already noticed by Blankenbecker et al. ${ }^{21}$ We have, therefore, opted for ignoring them altogether in the simulation rather than admitting them with positive weight. Although the situation is not entirely clear, especially for calculations in the grand canonical ensemble, it seems reasonable to exclude unphysical paths. See in this context Loh et al. ${ }^{26}$

\section{INTERPOLATING BETWEEN THE WEAK- AND THE STRONG-COUPLING REGIME}

According to the Mermin-Wagner theorem, ${ }^{27}$ infiniterange magnetic order is forbidden in two dimensions at any $T \neq 0$. This is so because the Goldstone modes strongly disorder the system giving rise to a spin-spin correlation length, which decays with the temperature as $\xi(T) \sim \exp (A / T)$, where $A$ is a constant. Thus no phase transitions of magnetic origin can take place in an infinite system except, perhaps, at $T=0$. Other kinds of phase transitions are outside the scope of this theorem. Such is the case, e.g., of the (Mott) paramagnetic metal-paramagnetic insulator transition. We now study the one-electron DOS and the spin-structure factor for the case of the triangular lattice.

\section{A. The one-electron DOS}

The one-electron DOS is given by

$$
N(\omega)=\frac{1}{N} \sum_{k} A(k, \omega)
$$

where $N$ is the number of lattice sites and $A(k, \omega)$, the spectral-weight function, is the imaginary part of the retarded one-electron Green's function. $A(k \omega)$ is to be obtained from the QMC data for the Matsubara Green's function $G_{k}(\tau)$ in the imaginary-time domain $G_{k}(\tau)$, given by

$$
G_{k}(\tau)=-\left\langle c_{k}(\tau) c_{k}^{\dagger}(0)\right\rangle,
$$

where the brackets mean a grand canonical average, is antiperiodic in $0<\tau<\beta$ with $\beta=1 / T$. It can, therefore, be recast in Fourier series form ${ }^{28}$

$$
G_{k}(\tau)=\frac{1}{\beta} \sum_{n} e^{-i \omega \tau} G_{k}\left(i \omega_{n}\right)=-\int d \varepsilon \frac{A_{k}(\varepsilon)}{1+e^{-\beta \varepsilon}} e^{-\varepsilon \tau}
$$

with $\omega_{n}=(2 n+1)(\pi / \beta), n$ being any integer. The Fourier coefficients $G_{k}\left(i \omega_{n}\right)$ are easily shown to be

$$
G_{k}\left(i \omega_{n}\right)=\int d \varepsilon \frac{A_{k}(\varepsilon)}{i \omega_{n}-\varepsilon}
$$

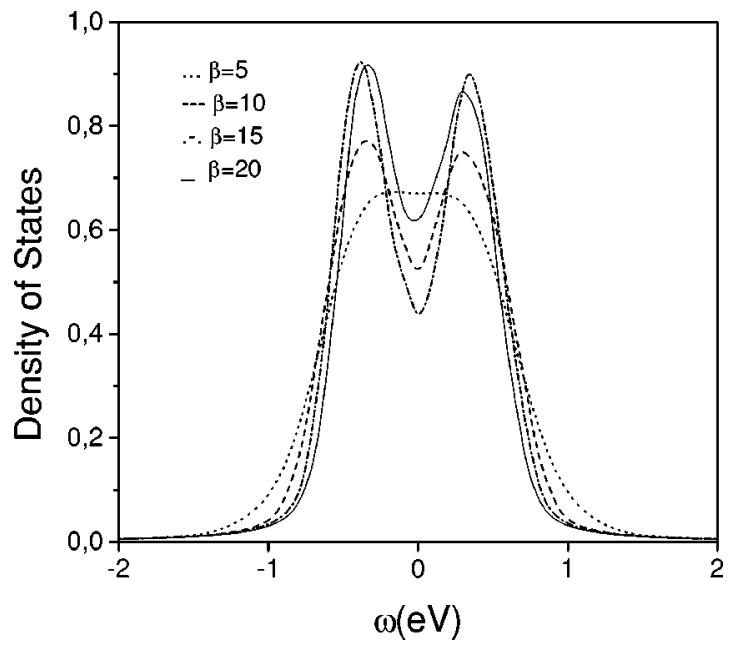

FIG. 4. One-electron density of states (DOS) of the triangular lattice for $U / t=5$ (weak coupling) and decreasing temperature, $\beta t=5,10,15$, and 20 .

i.e., just the Schmidt-Hilbert transform of $A_{k}(\varepsilon)$ whose analytic continuation along the real axis yields the retarded/ advanced Green's function $G_{k}(\omega+i \eta)$.

Despite its apparent simplicity, the numerical implementation of analytic continuations of this kind is, as a rule, difficult. One must invert either Eq. (6) or Eq. (7) in order to find $A_{k}(\varepsilon)$, an extremely ill-posed problem due to the statistical error in the QMC data for $G_{k}(\tau)$. Even small errors in $G_{k}(\tau)$ may be reflected in large changes in $A_{k}(\varepsilon)$. Any prior knowledge one may have about $A_{k}$ (e.g., sum rules, asymptotic behavior, etc.) helps somewhat to alleviate the situation and should be incorporated into a trial $A_{k}(\varepsilon)$, the default model, which ought to be as general as possible so as not to condition the final output very strongly. One must also ensure that the QMC data are as Gaussian distributed as possible. Finally Bayesian inference methods, such as the maximum-entropy principle must be applied in order to reject structure in $A_{k}(\varepsilon)$ not warranted by the data. In what follows, we use the annealing method described by Jarrel and Gubernatis. ${ }^{29}$

Going back to the triangular lattice, mean-field studies at $T=0$ (Ref. 30) have shown that the half-filled triangular lattice is a paramagnetic metal in the weak-coupling regime, in contrast with the SDW insulating character of the square lattice for small $U / t$. No gap in the one-electron DOS is, therefore, expected at any temperature for an infinite triangular lattice. It has been shown, however, that size effects are very strong in this regime. ${ }^{31} \mathrm{~A}$ gap in the one-electron DOS develops as soon as the spin-spin correlations extend over the whole system. Thus, for lattices of increasing size $N$ $\times N$, the system evolves from a situation where the correlation length $\xi(T)>N$, (with a gap) to one where $\xi(T)<N$ (without a gap). One should be careful when drawing conclusions about the existence of gaps from small lattices.

Figure 4 shows the one-electron DOS of a half-filled 4 $\times 4$ triangular lattice with periodic boundary conditions. We have taken $U / t=5$ ( $\sim$ half the bandwidth, $9 t)$, which is a weak-to-moderate value, and several values of $\beta, \beta t$ 


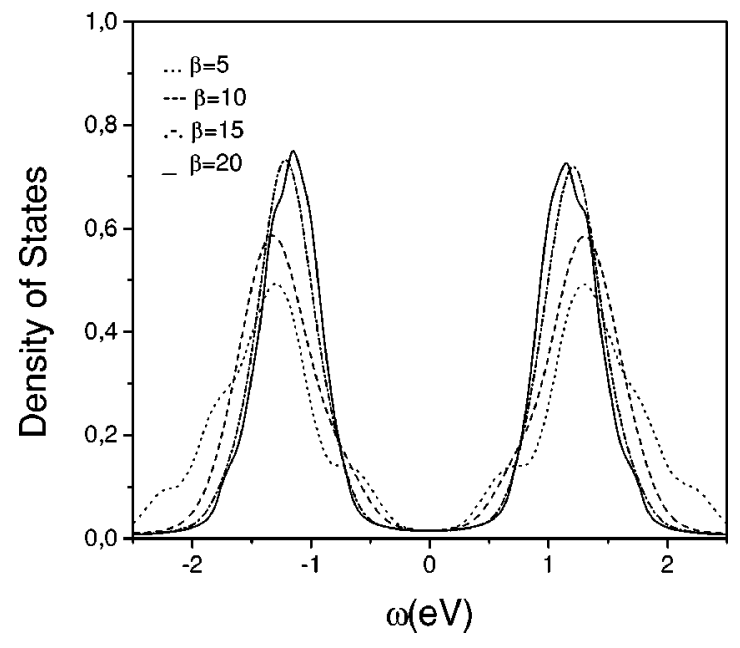

FIG. 5. Same as Fig. 4, but for $U / t=20$ (strong coupling).

$=5,10,15$, and 20. Even for $\beta t$ as high as 20, the system is far from having a fully developed gap. Since for the bigger lattices one expects weaker pseudogaps, it may be safely concluded that in the weak-coupling regime a triangular lattice has no gaps at any temperature, in accordance with the Mermin-Wagner theorem.

In the strong-coupling regime at $T=0$ the system is brought into a commensurate, three sublattice, $120^{\circ}$ twist SDW state (similar to the ground state of the classical antiferromagnet), which is insulating and stable for increasing $U$. Quantum fluctuations about the classical antiferromagnetic solution lead to the essential qualitative physics of the MottHubbard insulator at finite temperatures with a charge gap of order $U$ in the spectral-weight function. Figure 5 bears the same information as Fig. 4, but with $U / t=20$, which is deep inside the strong-coupling regime. Since size effects are very small in this regime, ${ }^{32}$ it is fairly clear that a fully developed gap is present at any temperature.

We thus see that, for a given temperature, the system evolves from a gapless situation at small $U$ to a fully developed gap at large $U$. As $U$ increases through the intermediate-coupling regime, one should find a critical value $U_{c}(T)$ for which the gap first appears. Figure 6 displays, as Figs. 4 and 5, the one-electron DOS for an intermediate value of $U / t=10$ ( $\sim$ the bandwidth). As the temperature is lowered from $\beta t=5$ down to $\beta t=20$, an incipient pseudogap gradually evolves into a fully developed gap. This value of $U$ is clearly below the critical $U$ for all $\beta t<20$, i.e., $U_{c}=10 t$ for $\beta t=20$. The complementary view is given in Fig. 7, which shows the one-electron DOS for $\beta t=5$ and $U / t=5,10,15$, and 20 . We see the system evolving from a gapless regime to a pseudogap, a deep pseudogap and finally a fully developed gap. Thus $U_{c}=20 t$ for $\beta t=5$. In this way one generates a temperature-dependent critical value of the coupling constant $U_{c}(T)$.

\section{B. The spin-structure factor}

The spin-structure factor $s(k)$ is given by the $k$-Fourier transform of the static spin-spin correlation function

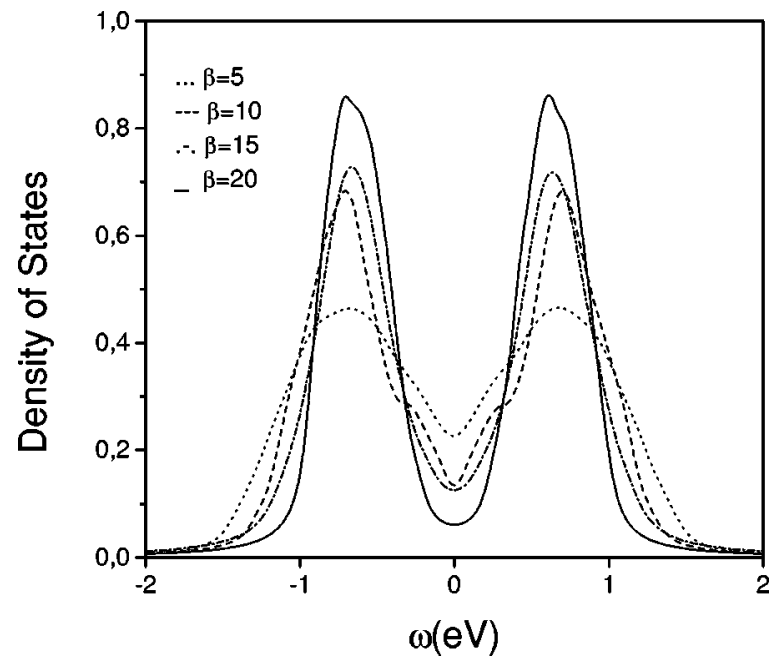

FIG. 6. Same as Figs. 4 and 5, but for $U / t=10$ (intermediate coupling).

$$
s_{i j}=\left\langle\sigma_{i z}(\tau) \sigma_{j z}(0)\right\rangle_{\tau=0^{+}}
$$

in the imaginary-time domain, where $\sigma_{i z}=n_{i \uparrow}-n_{i \downarrow}$. No analytic continuation is, therefore, required for the calculation of this quantity, since the dynamics has been integrated out when projecting onto zero (imaginary or real) time. The analytic continuation would be unavoidable, on the other hand, for extracting the dynamic (real frequency) spin susceptibility from QMC data for $\sigma_{i j}(\tau)$. The peaks of $s(k)$ and corresponding widths in $k$ space convey useful information about the spin ordering of the system and corresponding correlation length. Thus a sharp peak of $s(k)$ at $k=(\pi, \pi)$ on a square lattice indicates a long-range AF spin order, whereas a broad peak would indicate short-range order with the correlation length inversely proportional to the width of $s(\pi, \pi){ }^{33}$

Figure 8 shows $s(k)$ for $U / t=5,10$, and 20, representative values of the three coupling regimes, at $\beta t=20$, the lowest temperature we have considered in the present study. This figure should be taken in conjunction with Figs. 4-6 of the

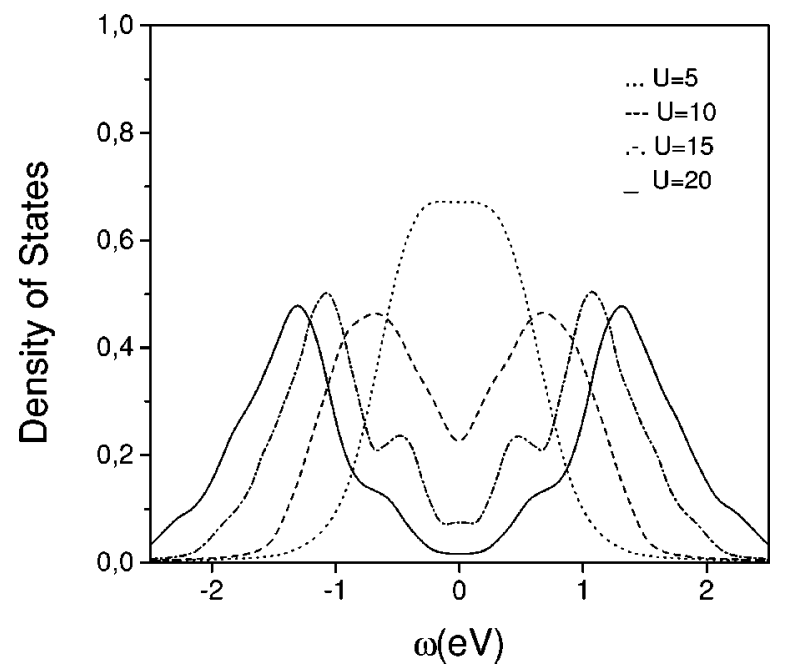

FIG. 7. One-electron DOS of the triangular lattice for increasing $U / t=5,10,15$, and 20 at a fixed temperature $\beta t=5$. 


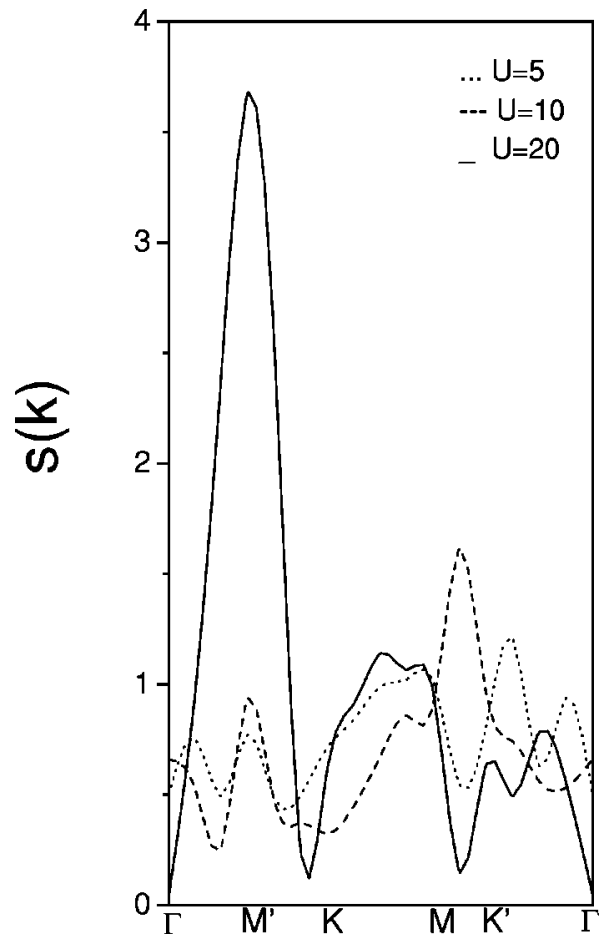

FIG. 8. Low-temperature $(\beta t=20)$ spin-structure factor $s(k)$ of the triangular lattice in the weak $(U / t=5)$, intermediate $(U / t$ $=10)$ and strong $(U / t=20)$ coupling regimes.

Sec. III A, which display the behavior of the one-electron DOS. Thus the gapsless DOS of Fig. 4 (weak coupling) for $\beta t=20$ is accompanied by an almost featureless $s(k)$ for $U / t=5$ on Fig. 8, indicating the lack of any spin order. We speak then of a spin-disordered gapless system (a paramagnetic metal). In the opposite, strong-coupling limit, one sees a fully developed gap in Fig. 5 in conjunction with a strong, sharp peak at the point $M^{\prime}=(2 \pi / 3,0)$ of the small SBZ, which is both very close to the Fermi surface at half filling, and a crossing point of the band structure associated with the three-sublattice model (bands $\varepsilon_{k}^{0}$ and $\varepsilon_{k}^{*}$ of Fig. 2). The spins, therefore, order into three sublattices with a twist angle of $120^{\circ}$, just the situation found in the strong-coupling regime of Sec. III A. As explained there, one then speaks of a Mott-Hubbard insulator.

The intermediate-coupling regime is somewhat more complex, as usual. Figure 8 shows two rather broad maxima centered about $M^{\prime}$ (small SBZ) and $M$ (large SBZ), both close to the Fermi surface. The spins are trying to arrange themselves in two different orderings driven by $M$ and $M^{\prime}$.
Beyond a critical $U_{c}(T)$ both spin orders collapse and the whole system becomes ordered, albeit in two domains. The one-electron DOS, as Fig. 6 shows for $\beta t=20$, develops a gap.

\section{CONCLUDING REMARKS}

The variation of the one-electron DOS with both the temperature and the coupling constant seems a useful tool for the purpose of identifying the different regimes of a given system. For the special case of the half-filled repulsive Hubbard model on a triangular lattice, a good example of a frustrated 2D system, we have identified an intermediate, temperaturedependent coupling regime, characterized by a two-domain SDW structure, which interpolates smoothly between the weak-coupling (paramagnetic metal) and the strong-coupling (Mott-Hubbard insulator) regimes. As the temperature is lowered in this intermediate-coupling regime, the system evolves from metallic to insulating. Alternatively, a critical $U_{c}(T)$ exists beyond which this frustrated 2D system develops a gap. The same situation (i.e., a critical $U$ ) has been shown to apply for a frustrated half-filled Hubbard model in one dimension. ${ }^{34}$ Since there is also a critical $U$ for the frustrated infinite-dimensional half-filled Hubbard model, ${ }^{35}$ it seems reasonable to assume that there is a critical $U$ for the frustrated half-filled Hubbard model in any dimension.

We conclude with a comment on the $\sqrt{3} \times \sqrt{3}$ adlayer structures on group-fourth semiconductor surfaces. Although a close connection with the above model study is not claimed, these structures may constitute a physical realization of the three coupling regimes just described, $\mathrm{Sn} / \mathrm{Ge}, \mathrm{Pb} /$ $\mathrm{Ge}$, and $\mathrm{SiC}$ being examples of the weak-, intermediate-, and strong-coupling regimes, respectively. Despite the added complexity due to electron-phonon interactions and atomic relaxation of both adsorbate and substrate atoms, the model study carried out here provides a general framework for the study of those systems. Let us stress, finally, that our main result in this paper, i.e., the existence of a critical $U$ for the frustrated half-filled Hubbard model in two dimensions may be of relevance, not only for the above semiconducting interfaces, but also in the context of theories of hightemperature superconductivity as well as in the study of certain $2 \mathrm{D}$ polymers.

\section{ACKNOWLEDGMENT}

This work was supported by the DGICYT (Spain) Project No. PB98-0683.
*FAX: +34-(1)5854894.

Email address: refolio@imaff.cfmac.csic.es

${ }^{1}$ G.W. Wannier, Phys. Rev. 79, 357 (1950).

${ }^{2}$ J. Stephenson, J. Math. Phys. 5, 1009 (1964).

${ }^{3}$ P.W. Anderson, Mater. Res. Bull. 8, 153 (1973).

${ }^{4}$ H.F. Pen, J. van den Brink, D.I. Khomskii, and G.A. Sawatzky, Phys. Rev. Lett. 78, 1323 (1997).

${ }^{5}$ J.M. Campinelli, H.H. Weitering, E.W. Plummer, and R. Stumpf, Nature (London) 381, 398 (1996).
${ }^{6}$ A. Mascaraque, J. Avila, E.G. Michel, and M.C. Asensio, Phys. Rev. B 57, 14758 (1998).

${ }^{7}$ A. Goldoni, C. Cepek, and S. Modesti, Phys. Rev. B 55, 4109 (1997).

${ }^{8}$ J.M. Campinelli, H.H. Weitering, M. Bartkowiak, and E.W. Plummer, Phys. Rev. Lett. 79, 2859 (1997).

${ }^{9}$ A. Goldoni and S. Modesti, Phys. Rev. Lett. 79, 3266 (1997).

${ }^{10}$ G. Lelay, V.Y. Aristov, O. Boström, J.M. Layet, M.C. Asensio, J. 
Avila, V. Huttel, and A. Cricenti, Appl. Surf. Sci. 123-124, 440 (1998).

${ }^{11}$ A. Mascaraque, J. Avila, J. Alvarez, M.C. Asensio, S. Ferrer, and E.G. Michel, Phys. Rev. Lett. 82, 2524 (1999).

${ }^{12}$ J. Avila, A. Mascaraque, E.G. Michel, M.C. Asensio, J. Ortega, R. Perez, and F. Flores, Phys. Rev. Lett. 82, 442 (1999).

${ }^{13}$ H.H. Weitering, J.M. Campinelli, A.V. Malechko, J. Zhang, M. Bartkowiak, and E.W. Plummer, Science 285, 2107 (1999).

${ }^{14}$ L.I. Johansson, F. Owman, and P. Martensson, Surf. Sci. 360, L478 (1998); J.M. Tremlim, I. Forbeaux, V. Langlais, H. Belkir, and J.M. Debever, Europhys. Lett. 39, 61 (1997).

${ }^{15}$ H.H. Weitering, X. Shi, P.D. Johnson, J. Chen, N.J. Dinardo, and S. Kempa, Phys. Rev. Lett. 78, 1331 (1997).

${ }^{16}$ K. Würde, P. Krüger, A. Mazur, and J. Pollman, Surf. Rev. Lett. 5, 105 (1998).

${ }^{17}$ S. Scandolo, F. Ancilotto, G.I. Chiarotti, G. Santoro, S. Serra, and E. Tosatti, Surf. Sci. 402-404, 808 (1998).

${ }^{18}$ G. Santoro, S. Sorella, F. Becca, S. Scandolo, and E. Tosatti, Surf. Sci. 402-404, 802 (1998).

${ }^{19}$ G. Santoro, S. Scandolo, and E. Tosatti, Phys. Rev. B 59, 1891 (1999).

${ }^{20}$ V.I. Anisimov, A.F. Bedin, M.A. Korotin, G. Santoro, S. Scandolo, and E. Tosatti, Phys. Rev. B 61, 1752 (2000).
${ }^{21}$ R. Blankenbecker, D.J. Scalapino, and R.L. Sugar, Phys. Rev. D 24, 2278 (1981).

${ }^{22}$ J.E. Hisch, Phys. Rev. B 28, 4059 (1983).

${ }^{23}$ J.E. Hisch, Phys. Rev. B 31, 4403 (1985).

${ }^{24}$ S.R. White, D.J. Scalapino, R.L. Sugar, E.Y. Loh, J.E. Gubernatis, and R.T. Scalettar, Phys. Rev. B 40, 506 (1989).

${ }^{25}$ E.Y. Loh and J.E. Gubernatis, in Electronic Phase Transitions, edited by W. Hanke and Y.V. Kopaev (Elsevier, New York, 1992), Chap. 4.

${ }^{26}$ E.Y. Loh, J.E. Gubernatis, R.T. Scalettar, S.R. White, D.J. Scalapino, and R.L. Sugar, Phys. Rev. B 41, 9301 (1990).

${ }^{27}$ N.D. Mermin and H. Wagner, Phys. Rev. Lett. 17, 1133 (1966).

${ }^{28}$ L.P. Kadanoff and G. Baym, Quantum Statistical Mechanics (Benjamin, New York, 1962), Chap 3.

${ }^{29}$ M. Jarrel and J.E. Gubernatis, Phys. Rep. 269, 135 (1996).

${ }^{30}$ H.R. Krishnamurthy, C. Jayaprakash, Sanjoy Sarker, and Wolfgand Wenzel, Phys. Rev. Lett. 64, 950 (1990).

${ }^{31}$ S.R. White, Phys. Rev. B 46, 5678 (1992).

${ }^{32}$ S.R. White, Phys. Rev. B 47, 1160 (1993).

${ }^{33}$ A. Kampf and J.R. Schrieffer, Phys. Rev. B 41, 6399 (1990).

${ }^{34}$ R. Bulla, Phys. Rev. B 83, 136 (1999).

${ }^{35}$ A. Georges, G. Kotliar, W. Krauth, and M.J. Rozenberg, Rev. Mod. Phys. 68, 13 (1996). 